\title{
THEMIS observation of a substorm event on 04:35, 22 February 2008
}

\author{
J. Liu ${ }^{1}$, V. Angelopoulos ${ }^{1}$, H. Frey ${ }^{2}$, J. McFadden ${ }^{2}$, D. Larson ${ }^{2}$, K. Glassmeier ${ }^{3}$, S. Mende ${ }^{2}$, C. T. Russell ${ }^{1}$, I. J. Rae ${ }^{4}$, \\ K. R. Murphy ${ }^{4}$, and S. Apatenkov ${ }^{5}$ \\ ${ }^{1}$ IGPP/ESS University of California, Los Angeles, CA 90095-1567, USA \\ ${ }^{2}$ Space Sciences Laboratory, University of California, Berkeley, CA 94720-7450, USA \\ ${ }^{3}$ Institute for Geophysics and Extraterrestrical Physics, TUBS, Braunschweig, 38106, Germany \\ ${ }^{4}$ Department of Physics, University of Alberta, Edmonton, AB T6G 0A1, Canada \\ ${ }^{5}$ Institute of Physics, University of St. Petersburg, St. Petersburg, 198904, Russia
}

Received: 13 October 2008 - Revised: 2 April 2009 - Accepted: 2 April 2009 - Published: 4 May 2009

\begin{abstract}
We report on THEMIS in-situ and ground-based observations during a substorm between 04:30 04:50 UT on 22 February 2008. The spacecraft (probes) were aligned along the tail between $X_{\mathrm{GSM}}=-5 R_{E}$ to $-25 R_{E}$. The most distant probe $\mathrm{P} 1\left(X=-24.5 R_{E}\right)$ detected two successive tailward moving bipolar magnetic structures. $\mathrm{P} 2\left(X=-18 R_{E}\right)$, $\mathrm{P} 3\left(X=-11 R_{E}\right), \mathrm{P} 4\left(X=-10.5 R_{E}\right)$ all captured signatures related to the Earthward movement of a magnetic structure. THEMIS ground stations and all-sky imagers also recorded $\mathrm{Pi} 2$ pulsations and a sudden brightening in a white-light auroral imager followed by poleward expansion. We perform a detailed timing analysis of probe and ground-based data and reconstruct the time sequence of phenomena during this substorm. The earliest sign of substorm onset was the bipolar perturbation in the northward component of the magnetic field (interpreted as the result of reconnection onset) at P1 at 04:35:16 UT and corresponding magnetic perturbation at P2 at 04:35:14 UT. Auroral onset was seen at or before 04:36:55 UT, consistent with the visual onset of highlatitude magnetic pulsations at around that time. Earthward flows at P3 and P4 seen at $\sim 04: 36: 03 \mathrm{UT}$, and dipolarization onset at $\sim 04: 36: 50 \mathrm{UT}$, were observed at almost the same time as the ground onset signature, implying that near-Earth dipolarization happened in the aftermath of tail reconnection but not significantly ahead of the auroral intensification. Reconnection in the tail preceded ground onset and near-Earth dipolarization (current disruption) by $\sim 2 \mathrm{~min}$. Two reconnection pulses (the first one weaker than the second one) accompanied by correlative increases of cumulative magnetic flux transfer into the reconnection region were observed. A
\end{abstract}

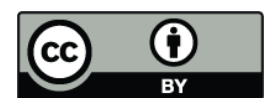

Correspondence to: J. Liu (jliu@igpp.ucla.edu) direct association of the reconnection pulses with two auroral intensifications can be made, suggesting that tail reconnection, like the auroral expansion, advances in steps rather than continuously.

Keywords. Magnetospheric physics (Magnetotail; Storms and substorms) - Space plasma physics (Magnetic reconnection)

\section{Introduction}

Substorms are global reconfigurations of the magnetosphere involving solar wind energy storage in Earth's magnetotail and abrupt conversion of energy to particle heating and kinetic energy (Akasofu, 1964; Axford, 1999). Substorms are responsible for various well documented magnetotail and ground phenomena: Satellites in the magnetotail at 15$30 R_{E}$ observe fast flows and magnetic field bipolar signatures in the $Z_{\mathrm{GSM}}$ and $X_{\mathrm{GSM}}$ direction, indicating Earthward or tailward moving plasmoids, or so-called nightside flux transfer events (NFTEs) (Sergeev et al., 1992) passing by. The sense of the bipolar signature depends on the location of the satellites relative to the reconnection site: positive-then-negative $B_{z}$ is observed for a tailward moving NFTE, whereas negative-then-positive $B_{z}$ is observed for an Earthward moving NFTE. It is expected that a fully formed NFTE can be observed far enough from the reconnection site. Closer to the reconnection site, a deflection of the tail field consistent with magnetic reconnection is expected, i.e., a northward deflection of the field Earthward of the reconnection site, and a southward deflection on the tailward side of the reconnection site. Near the neutral sheet, these magnetic deflections ought to be accompanied by flows away from the

Published by Copernicus Publications on behalf of the European Geosciences Union. 
reconnection site, whereas near the lobes these deflections are expected to be associated with flow towards the reconnection site, as predicted by typical reconnection topology. Closer to Earth, the reduction of the cross-tail current during substorms is observed as an increase in the Z-component of the magnetic field, often accompanied by a decrease in the X-component (Lopez and Lui, 1990), signifying that the field is changing from tail-like to dipole-like (a dipolarization). On occasion the large increase in $B_{z}$ is preceded by a small decrease in $B_{z}$, which has been attributed to an explosive build-up of the cross-tail current prior to its relaxation near substorm onset (Ohtani et al., 1992). One of the most defining aspects of a substorm is the auroral intensification and poleward expansion (Akasofu, 1964). The ground signatures of auroral substorms consist of rapid auroral intensification, breakup of auroral forms into smaller filaments, poleward expansion, and a westward surge of the most intense auroral arcs. Auroral activity is also associated with currents in the ionosphere. These currents cause ground magnetic signatures, including an abrupt increase of the auroral electrojet (AE) index and irregular magnetic field pulsations in the 40150 s range, called Pi2 pulsations (Jacobs et al., 1964; Saito, 1969).

Until very recently, the substorm onset mechanism was still in dispute. There are two main models for substorms: the current sheet disruption model (Lui, 1996) and the nearEarth neutral line model (Baker et al., 1996). The current disruption model asserts that when the current sheet thins, the ions become non-adiabatic and begin to stream across the current sheet in serpentine orbits and interact with adiabatic electrons drifting in the opposite direction, producing low-frequency whistler-type waves at $\sim 10 R_{E}$. These waves cause the plasma sheet to act resistively, thereby disrupting the cross-tail current. The process produces a rarefaction wave that propagates down the tail, which, in turn causes the typically observed reconnection signatures at 20$30 R_{E}$. The near-Earth neutral line model postulates that reconnection occurring at the current sheet at 20-30 $R_{E}$ (Nagai et al., 1998, 2005; Ge and Russell, 2006) is destabilized by either an external (solar wind) or internal (magnetotail) process. Reconnection produces Earthward and tailwardmoving flows. The Earthward flows interact with the nearEarth region producing intense field-aligned currents (Shiokawa et al., 1997; Birn et al., 1999; Zhang et al., 2007), which are then responsible for auroral intensification. The current disruption at $10 R_{E}$ and reconnection at 20-30 $R_{E}$ are connected by waves, and the communication time between these regions is on the order of $60 \mathrm{~s}$. The communication time to the ionosphere from either location is between $60-120 \mathrm{~s}$.

The THEMIS mission (Angelopoulos, 2008; Sibeck and Angelopoulos, 2008) was designed to address the question of which mechanism of substorm onset is supported by the data and how the different parts of the magnetosphere are connected causally. It employs five satellites (probes) on or- bits enabling them to align parallel to the Sun-Earth line once per four days. Thus, they can monitor tail phenomena simultaneously between $\sim 10 R_{E}$ and $\sim 20-30 R_{E}$ downtail. A network of ground-based observatories (GBOs) is also deployed to determine the meridian and onset time of Pi2 pulsations and auroral intensification (Mann et al., 2008; Mende et al., 2008; Russell et al., 2008). Here we present the timing results for a substorm that occurred on 22 February 2008. The event was first briefly reported in the on-line materials and methods section of Angelopoulos et al. (2008) and is similar to other events presented in that paper, as well as the event from Runov et al. (2008). In all these events auroral intensification was observed in the aftermath of magnetic reconnection onset, and preceded or happened at approximately the same time as current disruption onset. This paper presents significantly more information on the event and emphasizes the following aspects in the observations: (1) It shows that the particle anisotropy and flows at $\mathrm{P} 3$ and $\mathrm{P} 4$ are consistent with the Earthward motion of the plasma and cannot be interpreted as due to local relaxation of the plasma sheet. Specifically, the dipolarization onset is seen in the aftermath of the Earthward flow at $10 R_{E}$ and is likely due to the reconnection process further downtail. (2) It shows tail reconnection proceeds in a step-like fashion that can have a one-to-one correspondence to auroral activations, strengthening the aforementioned recent findings of a direct association between mid-tail reconnection and auroral activations during the early stages of substorm expansion.

\section{Instrumentation}

THEMIS, launched on 17 February 2007, consists of five identical satellites (probes) equipped with comprehensive particles and fields instrumentation (Angelopoulos, 2008). From December 2007 to April 2008, the five probes traveled through the magnetotail with different apogees: Probe 1 (P1) had an apogee of $\sim 30 R_{E}, \mathrm{P} 2$ of $\sim 20 R_{E}, \mathrm{P} 3$ and 4 of $\sim 12 R_{E}$, and $\mathrm{P} 5$ of $\sim 10 R_{E}$. (Note that the probe names are also given in letters: B, C, D, E, A corresponding to $1,2,3,4,5$, i.e., the letter sequence matches the number sequence after cyclical permutation by one letter position). The probe apogees and active control of in-orbit phasing enable probe observations of the same event in alignment at various altitudes; such alignments are timed to be between 00:00 and 12:00 UT, when the North American continent is over the nightside. There, the 20 THEMIS ground-based observatories (GBOs) (Mende et al., 2008), deployed from eastern Canada to western Alaska (several of these stations are part of the CARISMA (http://www.carisma.ca (Mann et al., 2008)) and GIMA (http://magnet.gi.alaska.edu/) magnetometer arrays) provide all-sky aurora imagers and ground magnetic field data. An additional 11 Education and Public Outreach (EPO) magnetometers (Russell et al., 2008) deployed across the United States complement the ground 


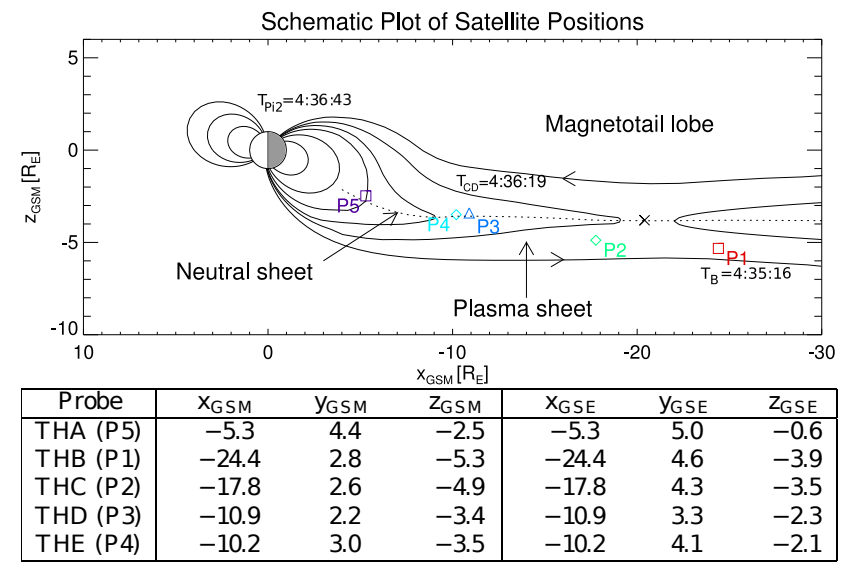

Fig. 1. Projections of THEMIS probes in $X-Z_{\mathrm{GSM}}$ plane along with representative field lines and neutral sheet location in GSM coordinates at $\sim 04: 36 \mathrm{UT}$.

magnetometer array with sub-auroral and mid-latitude observations. These observatories help determine the time of auroral intensification onset, negative bays and ULF wave onset.

In this paper we used THEMIS probe data from the Fluxgate Magnetometer (FGM) instrument (Auster et al., 2008), Solid State Telescope (SST) instrument which measures 25$50 \mathrm{keV}$ particles and Electro-Static Analyzer (ESA) instrument (McFadden et al., 2008) which measures 0-25 keV particles on the THEMIS probes. We also used All-Sky Imagers (ASI) (Mende et al., 2008) and Ground Fluxgate Magnetometers (GMAGs) (Russell et al., 2008). Finally, we used ground magnetometer data from mid-latitude INTERMAGNET network for contextual observations of ring current, partial ring current and substorm current wedge intensities.

\section{Timing}

\subsection{Timing ground signature onset}

The positions of the THEMIS probes from 04:00 05:00 UT on 22 February 2008 are shown in Fig. 1 and the THEMIS GBO positions are shown in Fig. 2. In Fig. 2 we also project probes P1-P5 using the Tsyganenko (1989) magnetospheric model along magnetic field lines to the ionosphere. The magnetic footprints of P1 to P4 are aligned from north to south near the east coast of Hudson Bay and P5 is near Rabbit Lake (Geomagnetic Lat. $\sim 66 \mathrm{~N}$, Long. $\sim 44 \mathrm{~W}$ ). The GBOs observed geomagnetic perturbations and auroral brightenings.

Using mid-latitude data from the INTERMAGNET network and several stations from THEMIS GBO segment we can fit model parameters of the substorm current wedge (SCW) (McPherron, 1979) and the ring current (DR). The free parameters in the model are: SCW magnitude and longitudinal positions of upward/downward field-aligned cur-

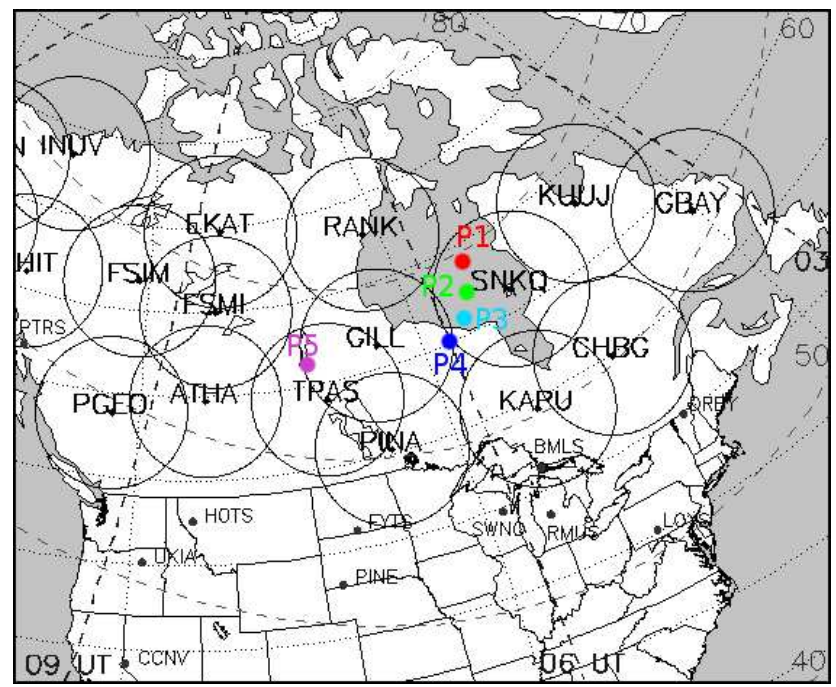

Fig. 2. Map of THEMIS ground observatories and the satellites' footprints mapping on the ionosphere at $\sim 04: 36 \mathrm{UT}$.

rents and DR magnitude. The current system models and the method are similar to those used by Horning et al. (1976) and Sergeev et al. (1996), but we have advantage in ground magnetometer coverage as compared to previous works. The result is shown in Fig. 3. Figure 3c, d shows the western and eastern edge of the substorm current wedge respectively. At 04:30 04:45 UT the substorm current wedge was localized around 23:00 MLT and had a width of $4 \mathrm{~h}$ of MLT. The five horizontal dashed lines indicate the projections of the five satellites THA-E (P5, P1-P4) to the ionosphere using the Tsyganenko (1989) model. It is evident that at $\sim 04: 35$ UT the footprints of the four outer probes (TH-B, -C, -D, -E, or $\mathrm{P} 1,2,3,4)$ were near the center of the SCW, meaning that the probes were well positioned to catch the space signatures of substorm onset in the magnetotail.

Figure 4 shows the observations of the geomagnetic field data and auroral intensity by the THEMIS GBOs as a function of time. The magnetic field data from high-latitude stations Gillam (Magnetic Latitude: 66.502 N, Longitude: $28.043 \mathrm{~W}$ ) and Sanikiluaq (Magnetic Latitude: 66.895 N, Longitude: $3.466 \mathrm{~W}$ ) which were closest to the meridian of the substorm are shown in Fig. 4b, c. Inspected by the naked eye, the magnetic perturbations at Sanikiluaq started at $\sim 04: 36: 43$ UT (denoted by dashed vertical line in Fig. $4 \mathrm{~b}$, c) and were coincident with small perturbations of $B_{X}$ and $B_{Y}$ at Gillam. Thus 04:36:43 UT is determined as the highlatitude Pi2 onset time.

For mid-latitude stations Ukiah, OR (Magnetic Latitude 51.317 N, Longitude 57.711 W), Pine Ridge, SD, Shawano, WI (Mag. Lat. 55.687 N, Lon. 18.191 W) and Remus, MI (Mag. Lat. 54.647 N, Lon. 12.992 W) seen in Fig. 4d-g, the magnetic field perturbation (sudden increase or decrease of $B_{Y}$ ) started at 04:37:49 UT (denoted by the vertical dashed 
(a)

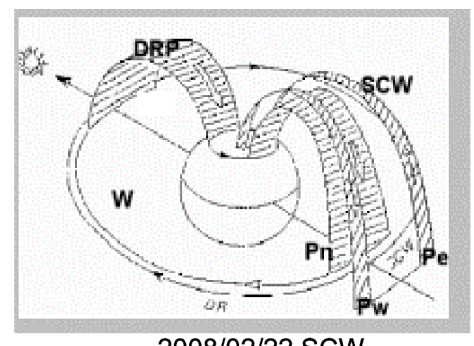

2008/02/22 SCW,

DR+SCW, 16 stations [-90..90sm] INTERMAGNET+THEMIS sbs\#1 baseline 04:30

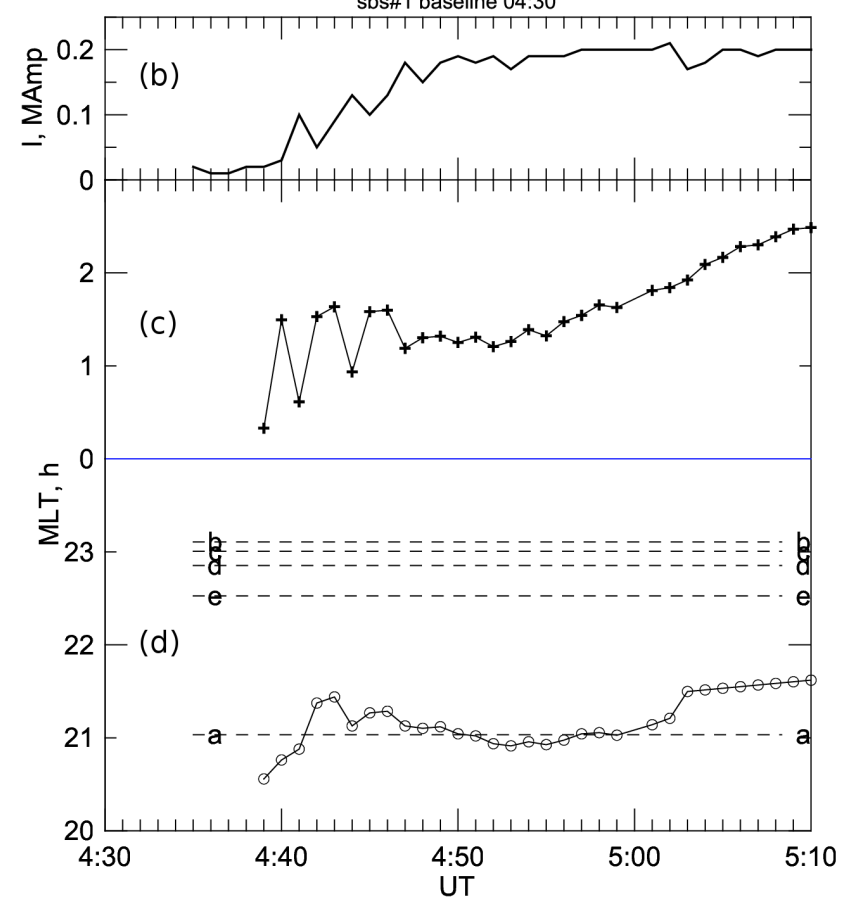

Fig. 3. Modeling mid-latitude ground magnetometer signatures to determine the central meridian, width and intensity of near-Earth space currents. Panels: (a) Pictorial representation of the model used (composed of line currents or series of line currents). (bd) Modeled intensity ( $\mathrm{mA}$ ) and longitude (degrees) of the current system components as a function of time past 04:30 UT, specifically: (b) SCW current intensity; (c, d) longitude of downward (crosses) and upward (circles) aligned currents, in SM-180 coordinates (i.e., Solar Magnetospheric longitude $-180^{\circ}$ ).

line in Fig. 4d-g), which is chosen to be the mid-latitude Pi2 onset time for this event. Although prior to this time $B_{X}$ had already began to increase (at $\sim 04: 36 \mathrm{UT}$ ), we interpret this as due to a growth phase enhancement of convection instead of Pi2 onset.

The location of the substorm current wedge (SCW) can also be determined by the ground magnetic field $B_{Y}$ (eastwest component) observation of mid-latitude stations Ukiah, Pine Ridge, Shawano, and Remus (aligned from west to east geographically, Fig. 2). At $\sim 04: 37: 49$ UT (the mid-latitude $\mathrm{Pi} 2$ onset time), all four stations (and many other stations, not shown here) observed gradual changes in $B_{Y}$, consistent

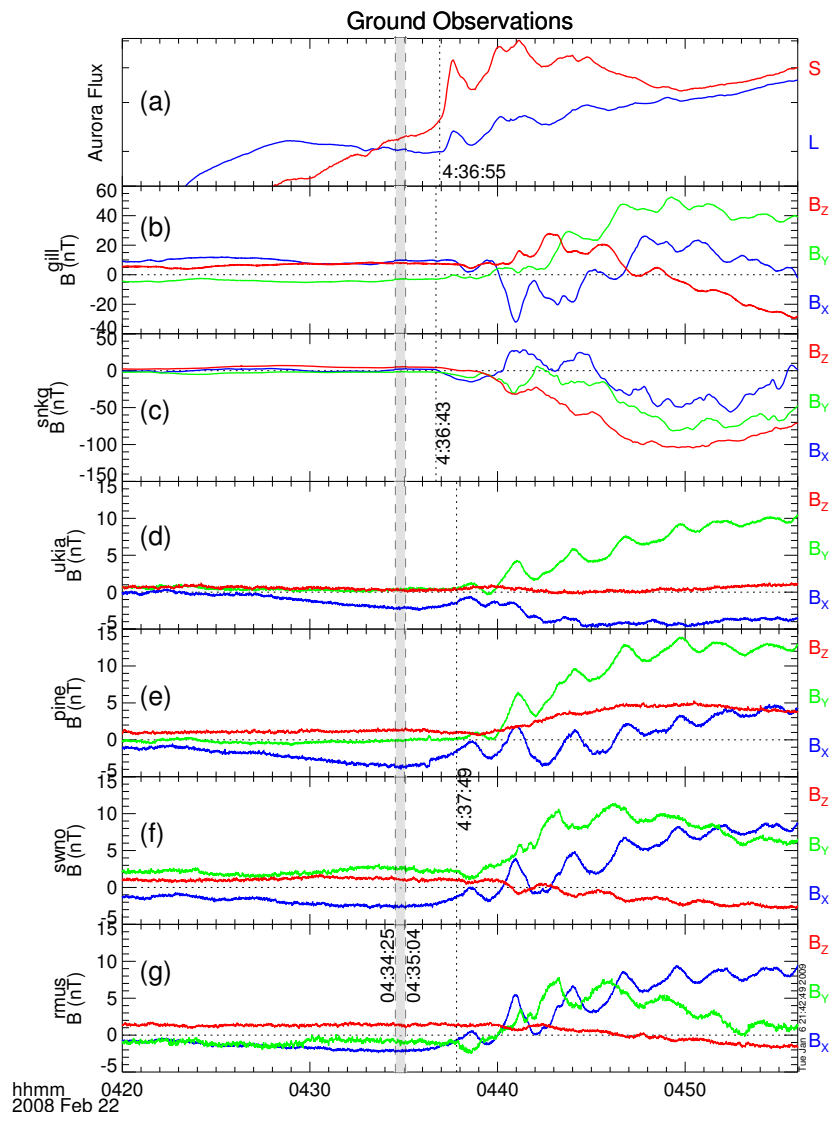

Fig. 4. Ground magnetometer observations of geomagnetic and aurora activities. Panels: (a) integrated light flux (rescaled, and in logarithm) of Gillam all-sky image (L blue area, S for the red area of Fig. 3); geomagnetic observation (median removed) of high-latitude (b) Gillam and (c) Sanikiluaq stations; mid-latitude (d) Ukiah, (e) Pine Ridge, (f) Shawano, and (g) Remus (aligned from West to East on the map) stations. The gray area denotes the range of possible tail reconnection onset time (discussed in Sect. 3.3).

with the onset of Pi2 pulsations. This change is positive at Ukiah and Pine Ridge, (and all stations west of Pine Ridge) but negative at Shawano and Remus (and all stations east of Shawano), both consistent with deflections from a large scale substorm current wedge centered between Pine Ridge (UT of midnight at 07:25 UT) and Shawano (UT of midnight at 06:05 UT). This is consistent with the location of the SCW determined by Intermagnet data in Fig. 3 .

The all-sky imager at Gillam captured the aurora intensification and expansion related to this event. A sequence of high-resolution all-sky images of Gillam from 04:36 UT to 04:43 UT is shown in Fig. 5. The integrated auroral intensity over the blue rectangle portion (Fig. 5) of the field of view at Gillam is shown as a blue trace in Fig. 4a. The integrated intensity over the most intense brightening within the red square in Fig. 5 at Gillam is also shown as a red trace in Fig. $4 a$, in arbitrary units on a linear scale. During the period of interest, Gillam had slightly cloudy skies, reflecting 

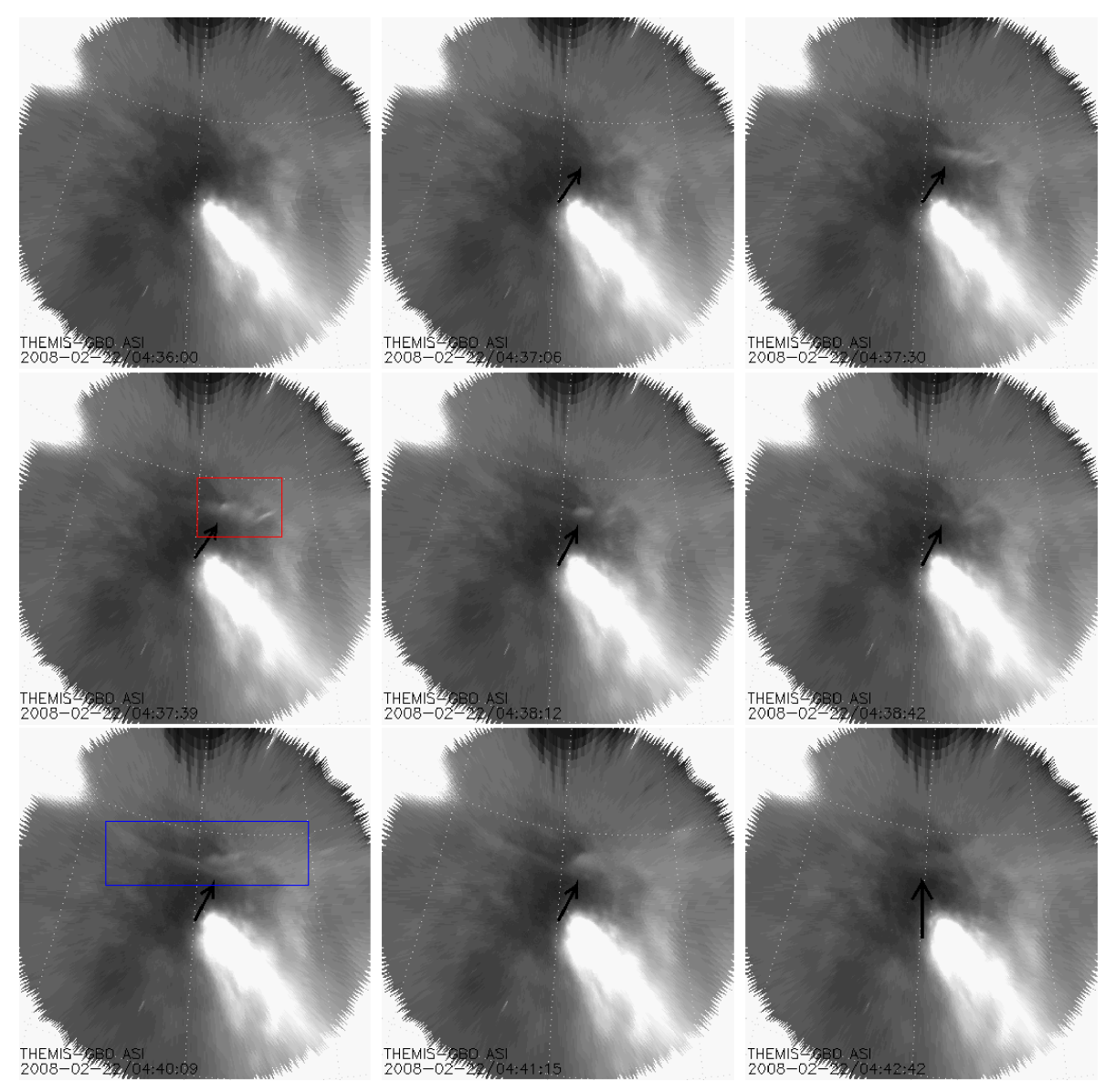

Fig. 5. All sky imagers' aurora photographs of the event at Gillam. Up is the direction of geographical north. The black arrow in the photographs points at the aurora arc. The blue and red square are the large $(\mathrm{L})$ and small $(\mathrm{S})$ integration area in Fig. $4 \mathrm{~b}$.

the moon from low at the southwest horizon and reflecting also at the northeast side of the dome. At $\sim 04: 36: 55$ UT the aurora began to intensify (dashed vertical line in Fig. 4a, determined as the inflection point of the red line). Then the arc began to expand westward and reached its maximum longitudinal extent of $\sim 400 \mathrm{~km}$ (the all-sky camera field of view is $\sim 800 \mathrm{~km}$ across the sky, mapped to $110 \mathrm{~km}$ in altitude in these images) and then died out at $\sim 04: 38: 30$ UT at a geomagnetic latitude of $69^{\circ}$. The arc began to expand westward again to a maximum width $\sim 400 \mathrm{~km}$ at $\sim 04: 38: 40$ UT and a new arc appeared and expanded poleward slightly at 04:39:21 UT. The arc continued moving poleward and westward until $\sim 04: 43$ UT. From Fig. $4 a$, it is clear that the aurora brightened at two major times, which is consistent with the two-step structure of the tail signatures which will be discussed later. The ground stations around Gillam all had cloudy skies, so the auroral onset was localized within the field of view of Gillam (Angelopoulos et al., 2008). Compared with typical substorm auroras, the aurora of this event is less dynamic, but it does exhibit both a brightening and a poleward expansion, which are important attributes of a substorm. The auroral intensification starting at 04:36:55 UT is consistent with the Pi2 onset at high-latitude Gillam, Sanikiluaq (04:36:43 UT). However, the onset of auroral intensification may have been masked by the thin clouds illuminated by the moon, and may have started a few frames earlier (images have 3-s cadence). Based on these observations, the auroral intensification and high-latitude $\mathrm{Pi} 2$ onsets are near-co-incidental to within a few 10 s of seconds, and represent the earliest determination of the substorm onset for this event.

The results from the optical observations above are consistent with the meridian of the substorm onset magnetic longitude, as determined from ground magnetometers. As shown in Fig. 2, the footprints of the THEMIS satellites are close to Gillam and within $1 \mathrm{~h}$ of MLT of the auroral onset location. This confirms that the THEMIS satellites were ideally positioned to examine the timing of substorm signatures in space.

\subsection{Timing magnetotail phenomena}

As shown in Fig. 1, all five probes are aligned along the magnetotail, from $X_{\mathrm{GSM}}=-5.3 R_{E}$ (near geosynchronous 


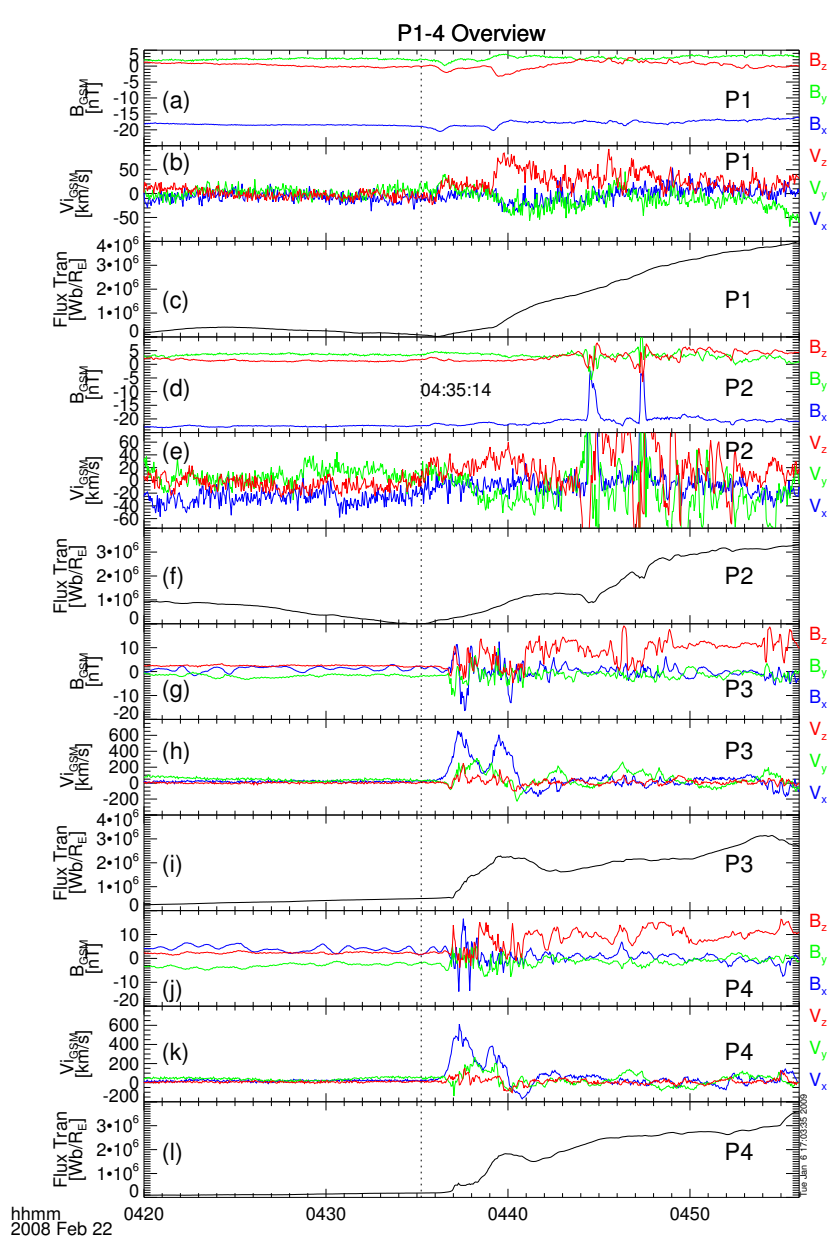

Fig. 6. Overview plot of P1, P2, P3 and P4. For each probe, from top to bottom: magnetic field in GSM coordinates; lowenergy (ESA) ion bulk velocity; cumulative magnetic flux transferred earthward and into the plasma sheet. The dashed vertical line across the whole height of the figure denotes the earliest observational tail signature (magnetic perturbation at P2) of the event.

altitude) to $-24.4 R_{E}$, and confined within $4 R_{E}$ in the $Z_{\mathrm{GSM}}$ direction, in a major conjunction.

Several key observables from probes P1, P2, P3 and P4 from 04:20 UT to 04:56 UT are shown in Fig. 6. At the position of $\mathrm{P} 1\left(X=-24.4 R_{E}\right)$ and $\mathrm{P} 2\left(X=-17.8 R_{E}\right)$, the magnetic field $B_{x}$ component is stable, negative and close to typical values near the plasma sheet boundary layer or lobe (Fig. 6a, d). Moreover, the peak differential energy flux of ions is around $2 \mathrm{keV}$ and the peak electron flux is at $\sim 100 \mathrm{eV}$ (P1 in Fig. 7d, e, P2 not shown). These are typical plasma sheet mean energies at that distance. Thus, P1 and P2 were inside the plasma sheet but close to its southern edge. P3 and P4 observed a magnetic field with $B_{x}$ and $B_{z}$ components that are on the order of $0-5 \mathrm{nT}$ and hovering around zero (Fig. $6 \mathrm{~g}, \mathrm{j}$ ). They also observed ions of $\sim 10 \mathrm{keV}$ and electrons of $\sim 1 \mathrm{keV}$ (P3 in Fig. $8 \mathrm{c}, \mathrm{d}, \mathrm{P} 4$ not shown) mean energies,. This means P3 and P4 were deep inside

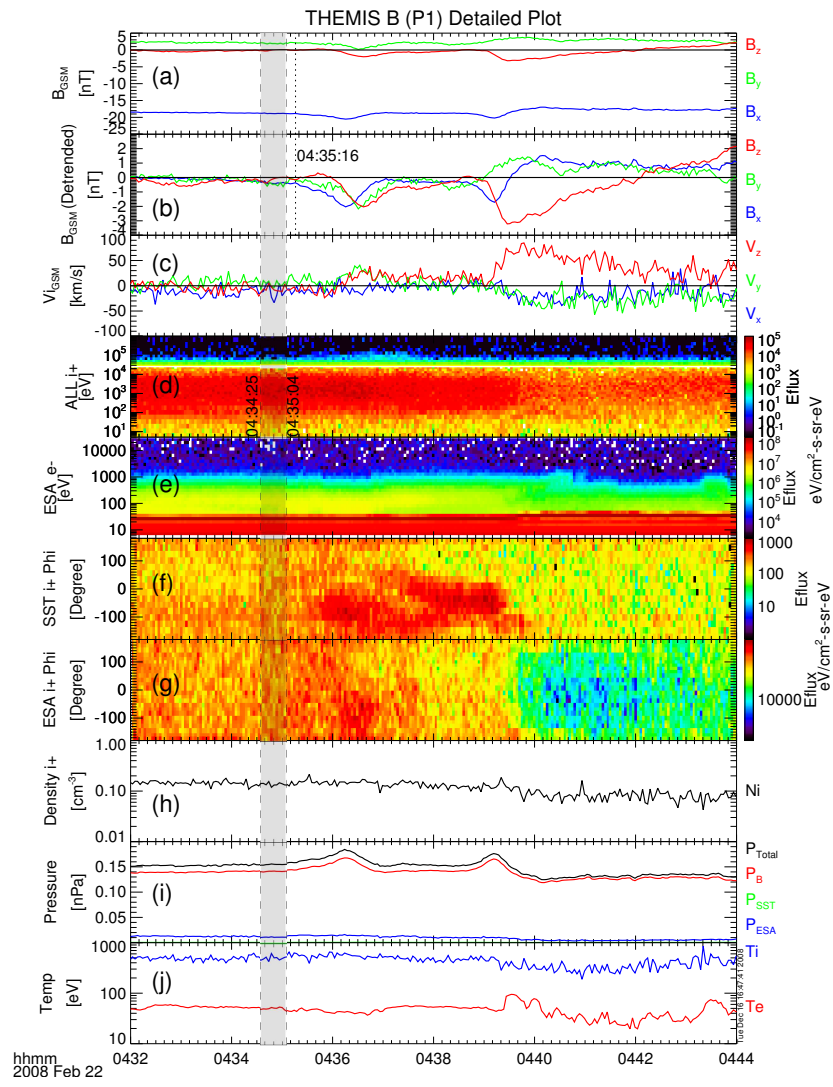

Fig. 7. Detailed data plot of P1 as a function of time. From top to bottom: (a) magnetic field in GSM coordinate; (b) magnetic field in GSM coordinate detrended; (c) low-energy (ESA) ion bulk velocity; (d) ions (ESA and SST combined) energy-eflux spectrum; (e) low energy electrons (ESA) energy-eflux spectrum; (f) Ion angular spectrogram in the phi direction $\left(0^{\circ}\right.$ : Earthward, $\pm 180^{\circ}$ : tailward, $90^{\circ}$ : dawnward, $-90^{\circ}$ : duskward, for this probe which is spinning right-handed with a spin axis near the ecliptic south) of the 25-50 keV ions from the SST instrument; (g) Same as in (f) but from the thermal ions of the ESA instrument; (h) Ion density; (i) Magnetic pressure, plasma pressure (ESA ion+electron and SST ion) and total pressure; (j) Ion and electron temperature in $\mathrm{eV}$. The gray area denotes the range of possible tail reconnection onset time (discussed in Sect. 3.3).

the plasma sheet, very close to the neutral sheet. Each of the five probes saw successive perturbations of the magnetic field (small perturbations on P1 and P2, large fluctuations on P3 and P4). Specifically, P1 saw two positive-then-negative $B_{z}$ bipolar signatures, consistent with two tailward-moving nightside flux transfer events (NFTEs) (Sergeev et al., 1992). P2 observed a small positive $B_{z}$ perturbation, consistent with the formation of reconnection topology at that location; flows $\left(V_{z}>0\right.$, peak $50 \mathrm{~km} / \mathrm{s}$, Fig. $6 \mathrm{~b}$, e) towards the neutral sheet which we interpret as inflow towards the reconnection site are consistent with that interpretation. P3 and P4 saw typical dipolarization signatures. The dashed line across all panels on Fig. 6 denotes the earliest tail signature of activity, which 
is the start of the magnetic perturbation on $\mathrm{P} 2$, and is within $2 \mathrm{~s}$ from the start of the magnetic perturbation at $\mathrm{P} 1$.

Figure $6 \mathrm{c}, \mathrm{f}, \mathrm{i}, 1$ shows the cumulative magnetic flux transfer into the plasma sheet or earthward (the integral of the $y$ component of $\left((-\boldsymbol{v} \times \boldsymbol{B})_{y}=v_{x} B_{z}-v_{z} B_{x}\right)$. Clearly on all four satellites the cumulative transferred magnetic flux started to increase significantly at the same time as the magnetic signature onset. Therefore, the cumulative transferred magnetic flux can also be used as a criterion of onset time here. P1 and P4 saw clear two-step development of the cumulative transferred magnetic flux, consistent with the two-step development of magnetic signatures and/or flow signatures observed by the satellites and the two-step brightenings of the aurora. The decrease of cumulative transferred magnetic flux before the onset time observed by $\mathrm{P} 1$ and $\mathrm{P} 2$ may be due either to data offset of velocity or a physical reason which requires further inspection.

Figure 7 presents detailed data as a function of time from $\mathrm{P} 1$, which observed tailward traveling NFTEs. Figure 7b shows the detrended magnetic field observation in GSM coordinates. The two successive magnetic field bipolar signatures are seen more clearly here, in Fig. 7b, than previously in Fig. 6a, in the detrended data from 04:35 UT to 04:40 UT. The northward-then-southward signature indicates two tailward traveling NFTEs. The first NFTE also caused an intensification of the $-90^{\circ}$ energetic ions in the angular spectrogram of the $30-80 \mathrm{keV}$ ions at $\sim 04: 35: 10$ UT. For P1, which is spinning with a spin axis near ecliptic south, the $-90^{\circ}$ spin phase angle corresponds to duskward moving particles. We interpret this as remote sensing of hot plasma sheet particles passing along with the NFTE tailward of P1, closer to the neutral sheet. After the center of the bipolar signature had passed, there is evidence of equatorward flow $\left(V_{z}>0\right.$, peak of $\sim 30 \mathrm{~km} / \mathrm{s}$ at 04:36:40 UT) at P1, which suggests equatorward flow of plasma sheet plasma into the reconnection site. A similar signature is observed after the passage of the second NFTE $(V z>0$, peak of $\sim 80 \mathrm{~km} / \mathrm{s}$ at 04:39:20UT).

In the aftermath of the second NFTE, there is a moderate reduction in plasma sheet ion density (from $\sim 0.11 \mathrm{~cm}^{-3}$ prior to $04: 39 \mathrm{UT}$ to $\sim 0.07 \mathrm{~cm}^{-3}$ at $\sim 04: 41 \mathrm{UT}$ seen in Fig. 7h) and ion and electron temperatures (about $10 \%$ reduction around the same time). This is also evidenced in the reduction in the plasma sheet differential energy fluxes seen around 04:40 UT in Fig. 7d. This suggests an reduction of the tail flux. At the same time the absolute value of the $B_{x}$ component of the magnetic field decreased, also indicating a reduction of the tail flux. The reduction of the tail flux is another signature supporting plasma sheet reconnection at that time. By 04:40 UT, the tailward particle motion, as evidenced in the ion flow velocity and tailward anisotropies of the angular spectrogram of the thermal ions in Fig. $7 \mathrm{~g}$ suggest that at this time reconnection reached the field lines at P1.

The total pressure (Fig. 7i) was also reduced in the aftermath of the second NFTE, primarily due to the reduction of

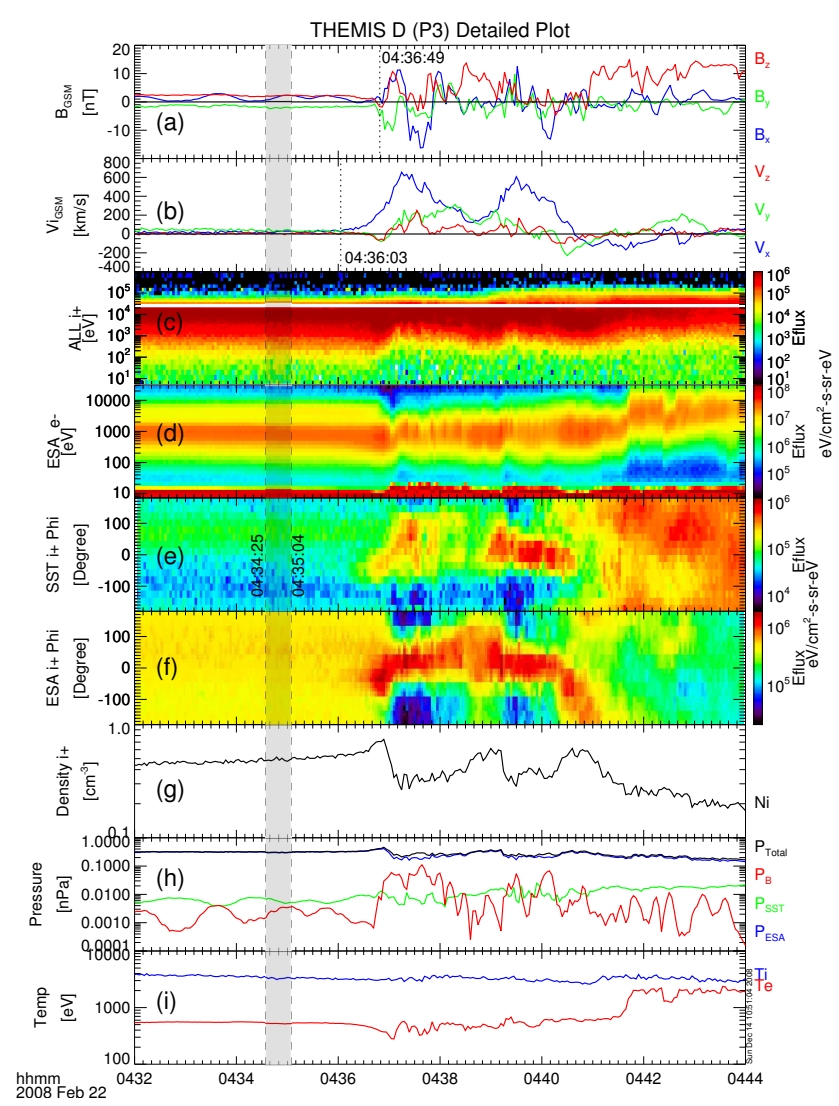

Fig. 8. Detailed data plot of $\mathrm{P} 3$ as a function of time. From top to bottom: (a) magnetic field in GSM coordinate; (b) low-energy (ESA) ion bulk velocity; (c) ions (ESA and SST combined) energyeflux spectrum; (d) low energy electrons (ESA) energy-eflux spectrum; (e-f) Ions phi-eflux spectrum $\left(0^{\circ}\right.$ : Earthward, $\pm 180^{\circ}$ : tailward, $90^{\circ}$ : duskward, $-90^{\circ}$ : dawnward, for this probe which is spinning right-handed with a spin axis near the ecliptic north); (g) ion density; (h) Magnetic pressure, plasma pressure (ESA ion + electron and SST ion) and total pressure. (i) Ion and electron temperature in $\mathrm{eV}$. The gray area denotes the range of possible tail reconnection onset time (discussed in Sect. 3.3).

the magnetic field. There was no significant plasma density change (Fig. 7h) at the time of the center of the NFTEs (but it decreased due to tail flux reduction at the end of the second NFTE) since P1 did not cut through the core of the NFTE, but the total pressure (plasma pressure including ESA ion and electron pressure and SST ion pressure plus magnetic pressure, Fig. 7i) peaked at $\sim 04: 36$ UT and $\sim 04: 39$ UT, the center-times of the NFTEs. During the time range of interest (04:32 UT to 04:44 UT), the plasma pressure was low ( $\sim 0.01 \mathrm{nPa}$, mainly ESA pressure, SST pressure is too small to be seen. Figure 7d illustrates why SST ( $>25 \mathrm{keV}$ particles) pressure is such smaller than ESA ( $<25 \mathrm{keV})$ particles.) and the total pressure is mainly magnetic pressure. This further confirms that P1 never entered the core area of the NFTEs and the pressure peaks are caused by the increasing of the 
Table 1. The time line of this event.

\begin{tabular}{lcc}
\hline Event & Time $(\mathrm{UT})$ & $X_{\mathrm{GSM}}\left(R_{E}\right)$ \\
\hline Reconnection onset & $04: 34: 25 \sim 35: 04$ & $-19.5 \sim-22.4$ \\
Reconnection effects at P2 & $04: 35: 14$ & -17.76 \\
Tailward NFTE onset at P1 & $04: 35: 16$ & -24.4 \\
Earthward flow onset at P3 & $04: 36: 03$ & -10.9 \\
High-latitude Pi2 onset & $04: 36: 43$ & -1 \\
Dipolarization on P3 & $04: 36: 49$ & -10.9 \\
Dipolarization at P4 & $04: 36: 50$ & -10.2 \\
Auroral intensification & $04: 36: 55$ & -1 \\
Mid-latitude Pi2 onset & $04: 37: 49$ & -1 \\
\hline
\end{tabular}

magnetic field in the vicinity of the NFTEs, and the increasing of the magnetic field is a result of the compression of the magnetotail plasma by the passing NFTE. We choose the beginning of the northward deflection of (detrended) magnetic field 04:35:16 UT as the first event onset (NFTE onset) time at P1 (shown as vertical dashed line in Fig. 7a, b).

Figure 8 shows detailed observations of P3, which was located at $X=\sim-11 R_{E}$ and was very close to the neutral sheet. P3 observed a series of dipolarizations starting at 04:36:49 UT (denoted by vertical dashed line in Fig. 8a). Please note that $B_{z}$ is quite small at this near-Earth location, which may be caused by a thin current sheet, and requires further inspection. P3 also captured the flow velocity of the two bulges of $\sim 600 \mathrm{~km} / \mathrm{s}$ which began at 04:36:03 UT and peaked at $\sim 04: 37: 10 \mathrm{UT}$ and $\sim 04: 39: 30 \mathrm{UT}$, respectively (Fig. 8b). At the time of these two peak flows the differential energy flux of energetic particles also increased (Fig. 8c). The ion angular spectra (Fig. 8e, f for $25 \sim 50 \mathrm{keV}$, and $5 \sim 25 \mathrm{keV}$ ions, respectively) show a higher flux of Earthward-moving particles $\left(\sim 0^{\circ}\right)$ than tailward particles $\left( \pm 180^{\circ}\right.$ ) at $\sim 04: 37 \mathrm{UT}$ and $\sim 04: 39: 30 \mathrm{UT}$, corresponding to the two flow peaks respectively. Note that as P3 is spinning with a spin axis near ecliptic north, phi $=90^{\circ}$ corresponds to duskward-moving particles. There are also duskward anisotropies ( $\mathrm{phi}=90^{\circ}$ ) before the onset of the flow which became larger at the time of the dipolarization. Ahead of the dipolarization at 04:36:49 UT, the particle density (Fig. 8g) and plasma pressure (Fig. 8h) increased. Because P3 was deep in the plasma sheet, the total pressure is mainly plasma pressure, but the magnetic pressure also increased from zero to $0.1 \mathrm{nPa}$ due to the dipolarization. We choose the start time of the Earthward flow above the background of $50 \mathrm{~km} / \mathrm{s}$ at 04:36:03 UT as the flow onset time of P3 (vertical dashed line in Fig. 8b).

The rise in ion density ahead of the dipolarization is consistent with the compression of the plasma ahead of the approaching flow. As reconnection involves particle populations from higher magnetic latitudes, which are less dense, we expect that reconnection results in rarefied flux tubes moving Earthward. This is consistent with previous obser- vations of high-speed flows (Angelopoulos et al., 1994) and is consistent with the lower density plasma observed at the time of dipolarization. Finally, it is important to note that the duskward energetic particle anisotropy prevails for $\sim 1$ min while an Earthward anisotropy is observed. The duskwarddrifting particles are the diamagnetic current carriers of the cross-tail current and their presence for several minutes prior to onset represents the stability of the thin current sheet prior to onset. The simultaneous presence of this anisotropic population along with the Earthward-drifting energetic particle component in Fig. 8e suggests that this new population comes from the tail and does not affect the local current sheet intensity and scale size. We therefore interpret the observed first onset of fast flows as due to reconnection-related compression of the plasma Earthward of the reconnection site.

P4 $\left(X=\sim-9.7 R_{E}\right.$ ) was very close to P3 so the observations at P4 are almost the same as P3. The flow onset at P4 was at 04:36:10 UT (Fig. 6k), i.e., a few seconds later than at P3 and the dipolarization time onset at P4 was at 04:36:50 UT (Fig. 6j), i.e., near-simultaneous to P3.

\subsection{Summary of timing}

Table 1 shows the times in which each probe saw the first related signature of the substorm. Our observations indicate that tail reconnection onset happened at or prior to 04:35:14 UT, between P1 and P3, and (based on the northward $B_{z}$ turning at P2) most likely tailward of P2. Based on the observations of P1 and P3, we can determine the location and onset time of reconnection, assuming for simplicity a constant speed of propagation of phenomena between P1 and P3.

Assuming that the initial perturbation is moving in constant speed (the magnetosonic speed in the plasma sheet), the reconnection site $x_{r}$ (GSM) can be obtained by solving the following equation with the NFTE/flow onset time of P1 and P3:

$\left(x_{3}-x_{r}\right)-\left(x_{r}-x_{1}\right)=V_{M S}\left(t_{3}-t_{1}\right)$,

where $x_{3}$ and $x_{1}$ are the positions of P3 and P1 in GSM coordinates; $t_{1}$ is the time when P1 captured the bipolar signature and $t_{3}$ is the Earthward flow onset time on P3 (as shown in Table 1 , we suppose a \pm 5 s error here), and $V_{M S}$ is the magnetosonic speed assumed constant between the two probes. The magnetosonic maximum speeds calculated from the observations at P1 and P3 are $\sim 610 \mathrm{~km} / \mathrm{s}$ and $\sim 1070 \mathrm{~km} / \mathrm{s}$ respectively, so we limit the possible propagation speed of the event to be between $610 \mathrm{~km} / \mathrm{s}$ and $1070 \mathrm{~km} / \mathrm{s}$. The site and time of reconnection determined with this method is shown in Fig. 9. The resultant reconnection site then is anywhere from $X=-19.5 R_{E}$ (corresponding to a signal propagation speed of $V_{M S}=610 \mathrm{~km} / \mathrm{s}$ ) to $X=-22.4 R_{E}$ (corresponding to a propagation speed of $V_{M S}=1070 \mathrm{~km} / \mathrm{s}$ ), tailward of $\mathrm{P} 2$. This result is consistent with our earlier interpretation that P2 saw an Earthward-moving structure. The time range of 
the reconnection onset is from 04:34:25 UT $\left(V_{M S}=610 \mathrm{~km} / \mathrm{s}\right)$ to 04:35:04 UT $\left(V_{M S}=1070 \mathrm{~km} / \mathrm{s}\right)$.

The reconnection onset time is determined to be about 2 min earlier than the high-latitude Pi2 onset at 04:36:43 UT and the Auroral Intensification onset at 04:36:55 UT. Considering the clouds over Gillam station which allows a possible earlier aurora onset time, the reconnection onset could be less than 2 min earlier than the ground signature onset. This is fast compared to the Alfvén propagation time from $20 R_{E}$ to the ionosphere, and this is similar to the observations reported for the 26 February 2008 substorm event (Angelopoulos et al., 2008). This possibly suggests that the waves launched in the equator may be kinetic, and thus may propagate faster than the MHD Alfvén speed (Lysak and Lotko, 1996).

The onset time of dipolarization at P3 and P4 at $X=\sim-10 R_{E}$ is $6 \mathrm{~s}$ and $7 \mathrm{~s}$ after the high-latitude Pi2 onset. If we interpret not just the flows but also the dipolarization front as a product of reconnection, this delay is explained as due to the time it takes for the flow emanating from the reconnection site to arrive at $\mathrm{P} 3$ and $\mathrm{P} 4$. This is measured to be $\sim 400 \mathrm{~km} / \mathrm{s}$ at the time of the dipolarization at P3. Assuming the pulse started at 04:34:32 UT at $x_{0}=20.3 R_{E}$, the magnetic perturbation arrival time at $\mathrm{P} 3$ is consistent with the observation speed of $400 \mathrm{~km} / \mathrm{s}$. Since the auroral intensification occurred nearly at the same time as the dipolarization onset at P3 and P4, it is not likely that the aurora was caused by the near-Earth flux pileup of reconnection flows, but rather by the reconnection activity further downtail.

\section{Conclusions and discussion}

The substorm event at $\sim 04: 35$ UT on 22 February 2008 has many characteristics that are similar to the event at 04:50 UT on 26 February 2008 presented by Angelopoulos et al. (2008) and the events presented by Gabrielse et al. (2009) and Runov et al. (2008). Specifically, we have shown that (1) tail reconnection, as evidenced by the release of a nightside flux transfer event at P1, was observed less than 2 min earlier than substorm onset as determined by auroral intensification or high latitude Pi2 pulsations and (2) that current disruption at $X=-10.9 R_{E}$ was observed almost at the same time as substorm onset, which implies that the currents associated with the local field dipolarization could not be the cause of the auroral intensification at substorm onset. In addition, we have shown that (3) the Earthward motion of the plasma prior to the dipolarization at P3 is consistent with the arrival of new particles from the tail, which likely originated in the region between P3 and the reconnection site, and (4) that the arrival time of the dipolarization at $\mathrm{P} 3$ is consistent with a release from the tail approximately at the time of onset. This suggests that the initial dipolarization observed at P3 is inconsistent with local disruption of the plasma, and is consistent with the arrival of magnetic flux from the tail. Finally, we have shown that (5) reduction of plasma sheet flux reached

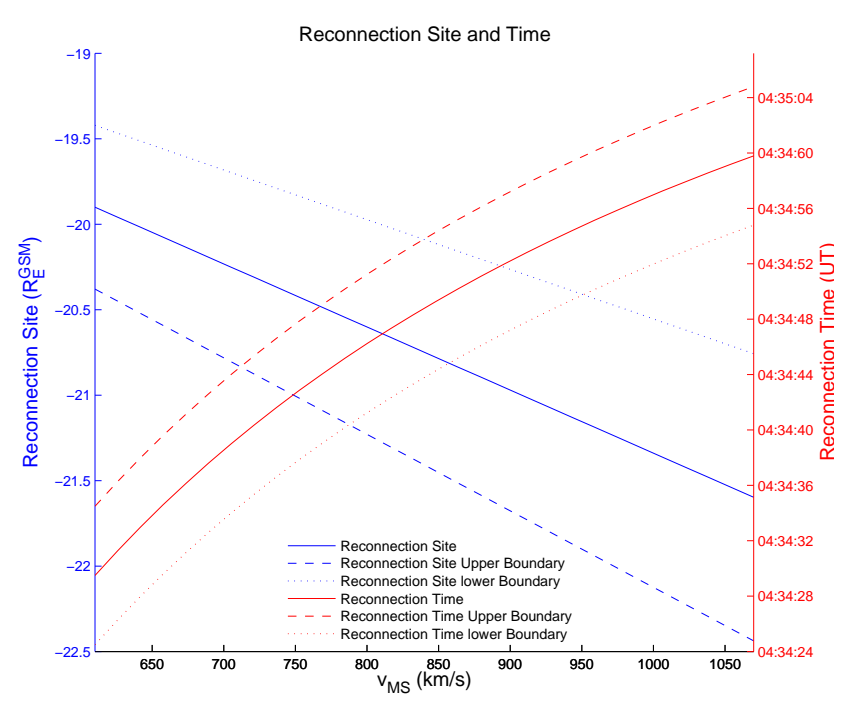

Fig. 9. Possible reconnection sites and time for different possible magnetosonic speed $V_{M S}$. Upper/Lower boundary is caused by the $\pm 5 \mathrm{~s}$ error of the time of the bipolar signature. Reconnection sites that are above the middle point between $\mathrm{P} 1$ and $\mathrm{P} 2$ are reasonable.

the location of probe $\mathrm{P} 1$ four minutes after the onset of reconnection signatures at that probe, and was likely accompanied by a reduction in the flux in the tail, consistent with the results of tail reconnection.

There were two NFTEs observed on P1, 3 min apart: the first resulted in only a minor reduction of the total pressure in its aftermath, whereas the second resulted in a $10 \%$ decrease in the total pressure. It is tempting to causally relate the two NFTEs (Fig. 7b, positive $B_{z}$ excursions at 04:35:16 UT and 04:38:00 UT) with the two Earthward flow pulses observed at $\mathrm{P} 3$ (Fig. 8b, positive $V_{x}$ excursions at 04:36:03 UT and 04:38:30 UT), two Earthward flow pulses (Fig. 6k, positive $V_{x}$ excursions at 04:36:03 UT and 04:38:40 UT) and two-step increase of cumulative transferred magnetic flux (Fig. 61) at P4, and the two auroral intensifications at Gillam (see Fig. 4a, 04:36:55 UT and 04:38:40 UT). Both NFTEs occurred prior to the auroral intensifications, and in both cases the flows started prior to or simultaneously to the auroral intensifications. It is interesting to note that while the former, weaker NFTE did not result in significant poleward expansion, the latter, stronger one resulted in substantial tail flux dissipation and significant poleward expansion.

These observations suggest that tail reconnection on occasion occurs in steps of increasing strength, and the results may be correlated to the intensity of auroral signatures and their poleward expansion. Despite the good correlation with the flows at P3, local measurements alone are not sufficient to determine the strength of reconnection and the amount of flux dissipation further downtail, or the auroral intensity and motion. This observation is consistent with the fact that the energy coupling of the reconnection process may be taking 
place by waves which emanate from higher latitudes than the equatorial plasma sheet at $10 R_{E}$.

Acknowledgements. THEMIS was made possible and is supported in the US by NASA contract NAS5-02099. Financial support for the FGM instrument was provided by the German Ministry for Economy and Technology and the German Center for Aviation and Space (DLR) under contract 50 OC 0302. CARISMA is operated by the University of Alberta and funded by the Canadian Space Agency. GIMA data is provided by the Geophysical Institute of the University of Alaska Fairbanks, and the Canadian Magnetic Observatory System (CANMOS) network, maintained and operated by the Geological Survey of Canada, also provided data used in this study.

Topical Editor R. Nakamura thanks L. Kepko and another anonymous referee for their help in evaluating this paper.

\section{References}

Akasofu, S.-I.: The development of the auroral substorm, Planet. Space Sci., 12, 273-282, doi:10.1016/0032-0633(64)90151-5, 1964.

Angelopoulos, V.: The THEMIS Mission, Space Sci. Rev., 141, 534, doi:10.1007/s11214-008-9336-1, 2008.

Angelopoulos, V., Kennel, C. F., Coroniti, F. V., Pellat, R., Kivelson, M. G., Walker, R. J., Russell, C. T., Baumjohann, W., Feldman, W. C., and Gosling, J. T.: Statistical characteristics of bursty bulk flow events, J. Geophys. Res., 99, 21257-21280, 1994.

Angelopoulos, V., McFadden, J. P., Larson, D., Carlson, C. W., Mende, S. B., Frey, H., Phan, T., Sibeck, D. G., Glassmeier, K.H., Auster, U., Donovan, E., Mann, I. R., Rae, I. J., Russell, C. T., Runov, A., Zhou, X.-Z., and Kepko, L.: Tail Reconnection Triggering Substorm Onset, 321, 931, doi:10.1126/science.1160495, 2008.

Auster, H. U., Glassmeier, K. H., Magnes, W., Aydogar, O., Baumjohann, W., Constantinescu, D., Fischer, D., Fornacon, K. H., Georgescu, E., Harvey, P., Hillenmaier, O., Kroth, R., Ludlam, M., Narita, Y., Nakamura, R., Okrafka, K., Plaschke, F., Richter, I., Schwarzl, H., Stoll, B., Valavanoglou, A., and Wiedemann, M.: The THEMIS Fluxgate Magnetometer, Space Sci. Rev., 141, 235-264, doi:10.1007/s11214-008-9365-9, 2008.

Axford, W.: Reconnection, substorms and solar flares, Physics and Chemistry of the Earth C, 24, 147-151, doi:10.1016/ S1464-1917(98)00022-1, 1999.

Baker, D. N., Pulkkinen, T. I., Angelopoulos, V., Baumjohann, W., and McPherron, R. L.: Neutral line model of substorms: Past results and present view, J. Geophys. Res., 101, 12975-13010, doi:10.1029/95JA03753, 1996.

Birn, J., Hesse, M., Haerendel, G., Baumjohann, W., and Shiokawa, K.: Flow braking and the substorm current wedge, J. Geophys. Res., 104, 19895-19904, doi:10.1029/1999JA900173, 1999.

Gabrielse, C., Angelopoulos, V., Runov, A., Frey, H. U., McFadden, J., Larson, D. E., Glassmeier, K.-H., Mende, S., Russell, C. T., Apatenkov, S., Murphy, K. R., and Rae, I. J.: Timing and localization of near-Earth tail and ionospheric signatures during a substorm onset, J. Geophys. Res., 114, 0, doi: 10.1029/2008JA013583, 2009.
Ge, Y. S. and Russell, C. T.: Polar survey of magnetic field in near tail: Reconnection rare inside $9 \mathrm{R}_{E}$, Geophys. Res. Lett., 33, 2101, doi:10.1029/2005GL024574, 2006.

Horning, B., McPherron, R., and Jackson, D.: Application of linear inverse theory to a line current model of substorm current systems, J. Geophys. Res., 79, 5202-5210, 1976.

Jacobs, J. A., Kato, Y., Matsushita, S., and Troitskaya, V. A.: Classification of Geomagnetic Micropulsations, J. Geophys. Res., 69, 180, 1964.

Kepko, L., Kivelson, M. G., McPherron, R. L., and Spence, H. E.: Relative timing of substorm onset phenomena, J. Geophys. Res., 109, 4203, doi:10.1029/2003JA010285, 2004.

Lopez, R. E. and Lui, A. T. Y.: A multisatellite case study of the expansion of a substorm current wedge in the near-earth magnetotail, J. Geophys. Res., 95, 8009-8017, 1990.

Lui, A. T. Y.: Current disruption in the Earth's magnetosphere: Observations and models, J. Geophys. Res., 101, 13067-13088, doi: 10.1029/96JA00079, 1996.

Lysak, R. L. and Lotko, W.: On the kinetic dispersion relation for shear Alfvén waves, J. Geophys. Res., 101, 5085-5094, doi:10. 1029/95JA03712, 1996.

Mann, I. R., Milling, D. K., Rae, I. J., Ozeke, L. G., Kale, A., Kale, Z. C., Murphy, K. R., Parent, A., Usanova, M., Pahud, D. M., Lee, E.-A., Amalraj, V., Wallis, D. D., Angelopoulos, V., Glassmeier, K.-H., Russell, C. T., Auster, H.-U., and Singer, H. J.: The Upgraded CARISMA Magnetometer Array in the THEMIS Era, Space Sci. Rev., 141, 413-451, doi: 10.1007/s11214-008-9457-6, 2008.

McFadden, J. P., Carlson, C. W., Larson, D., Bonnell, J., Mozer, F., Angelopoulos, V., Glassmeier, K.-H., and Auster, U.: THEMIS ESA First Science Results and Performance Issues, Space Sci. Rev., 141, 477-508, doi:10.1007/s11214-008-9433-1, 2008.

McPherron, R. L.: Magnetospheric substorms., Rev. Geophys. Space Phys., 17, 657-681, 1979.

Mende, S. B., Harris, S. E., Frey, H. U., Angelopoulos, V., Russell, C. T., Donovan, E., Jackel, B., Greffen, M., and Peticolas, L. M.: The THEMIS Array of Ground-based Observatories for the Study of Auroral Substorms, Space Sci. Rev., 141, 357-387, doi:10.1007/s11214-008-9380-x, 2008.

Nagai, T., Fujimoto, M., Saito, Y., Machida, S., Terasawa, T., Nakamura, R., Yamamoto, T., Mukai, T., Nishida, A., and Kokubun, S.: Structure and dynamics of magnetic reconnection for substorm onsets with Geotail observations, J. Geophys. Res., 103, 4419-4440, doi:10.1029/97JA02190, 1998.

Nagai, T., Fujimoto, M., Nakamura, R., Baumjohann, W., Ieda, A., Shinohara, I., Machida, S., Saito, Y., and Mukai, T.: Solar wind control of the radial distance of the magnetic reconnection site in the magnetotail, J. Geophys. Res., 110, 9208, doi:10.1029/ 2005JA011207, 2005.

Ohtani, S., Takahashi, K., Zanetti, L. J., Potemra, T. A., McEntire, R. W., and Iijima, T.: Initial signatures of magnetic field and energetic particle fluxes at tail reconfiguration - Explosive growth phase, J. Geophys. Res., 97, 19311-19324, 1992.

Runov, A., Angelopoulos, V., Zhou, X.-Z., Voronkov, I. O., Kubyshkina, M. V., Nakamura, R., Carlson, C. W., Frey, H. U., McFadden, J., Larson, D., Mende, S. B., Glassmeier, K.-H., Auster, U., and Singer, H. J.: Multipoint in situ and groundbased observations during auroral intensifications, J. Geophys. Res., 113, 0, doi:10.1029/2008JA013493, 2008. 
Russell, C. T., Chi, P. J., Dearborn, D. J., Ge, Y. S., Kuo-Tiong, B., Means, J. D., Pierce, D. R., Rowe, K. M., and Snare, R. C.: THEMIS Ground-Based Magnetometers, Space Sci. Rev., 141, 389-412, doi:10.1007/s11214-008-9337-0, 2008.

Saito, T.: Geomagnetic Pulsations, Space Sci. Rev., 10, 319-412, doi:10.1007/BF00203620, 1969.

Sergeev, V. A., Elphic, R. C., Mozer, F. S., Saint-Marc, A., and Sauvaud, J. A.: A two satellite study of nightside flux transfer events in the plasma sheet, Planet. Space Sci., 40, 1551-1572, doi:10.1016/0032-0633(92)90052-P, 1992.

Sergeev, V. A., Vagina, L. I., Elphinstone, R. D., Murphree, J. S., Hearn, D. J., Cogger, L. L., and Johnson, M. L.: Comparison of UV optical signatures with the substorm current wedge as predicted by an inversion algorithm, J. Geophys. Res., 101, 26152628, doi:10.1029/95JA00537, 1996.
Shiokawa, K., Baumjohann, W., and Haerendel, G.: Braking of high-speed flows in the near-Earth tail, Geophys. Res. Lett., 24, 1179-1182, doi:10.1029/97GL01062, 1997.

Sibeck, D. G. and Angelopoulos, V.: THEMIS Science Objectives and Mission Phases, Space Sci. Rev., 141, 35-59, doi:10.1007/ s11214-008-9393-5, 2008.

Tsyganenko, N. A.: A magnetospheric magnetic field model with a warped tail current sheet, Planet. Space Sci., 37, 5-20, doi: 10.1016/0032-0633(89)90066-4, 1989.

Zhang, H., Pu, Z. Y., Cao, X., Fu, S. Y., Liu, Z. X., Ma, Z. W., Dunlop, M. W., Baumjohann, W., Xiao, C. J., Hong, M. H., Cao, J. B., Zong, Q. G., Wang, X. G., Carr, C., Rème, H. A., Dandouras, I., Fazakerley, A., Frey, H. U., and Escoubet, C. P.: TC-1 observations of flux pileup and dipolarization-associated expansion in the near-Earth magnetotail during substorms, Geophys. Res. Lett., 34, 3104, doi:10.1029/2006GL028326, 2007. 